TRANSACTIONS OF THE

AMERICAN MATHEMATICAL SOCIETY

Volume 349, Number 12, December 1997, Pages 5093-5105

S $0002-9947(97) 01913-2$

\title{
ON A GENERAL FORM OF THE SECOND MAIN THEOREM
}

\author{
MIN RU
}

\begin{abstract}
We give a proof of a general form of the Second Main Theorem for holomorphic curves with a good error term. Two applications of this general form are also provided.
\end{abstract}

\section{INTRODUCTION}

In this paper, we give a proof of a general form of the Second Main Theorem for holomorphic curves in Nevanlinna theory (Theorem 2.3) by using the techniques of Ye [19] to get a good error term. This theorem was first proved by Vojta [15], but with a less precise error term. We then explain why this general form is more useful than the usual form of the Second Main Theorem by giving two applications. The first application is a simple proof of the Second Main Theorem with moving targets, originally obtained by $\mathrm{Ru}$ and Stoll [8]. We indicate that the general form of the SMT with fixed hyperplanes (Theorem 2.1) implies the Second Main Theorem with moving targets. The second application is to establish a Second Main Theorem for linearly degenerate holomorphic mappings with a good error term. We note that all the proofs in this paper work also when the domain is $\mathbf{C}^{m}$ instead of $\mathbf{C}$.

\section{A GENERAL FORM OF THE SECOND MAIN THEOREM WITH A GOOD ERROR TERM}

Motivated by Schmidt's subspace theorem in number theory, Vojta [15] formulated the following general form of the Second Main Theorem for holomorphic curves. Here we use the standard notation in Nevanlinna theory.

Theorem 2.1 (A general form of the SMT). Let $f=\left[f_{0}: \cdots: f_{n}\right]: \mathbf{C} \rightarrow \mathbf{P}^{n}(\mathbf{C})$ be a holomorphic curve whose image is not contained in any proper subspaces. Let $H_{1}, \ldots, H_{q}$ be arbitrary hyperplanes in $\mathbf{P}^{n}(\mathbf{C})$. Then, for every $\epsilon>0$,

$$
\int_{0}^{2 \pi} \max _{K} \sum_{j \in K} \lambda_{H_{j}}\left(f\left(r e^{i \theta}\right)\right) \frac{d \theta}{2 \pi} \cdot \leq \cdot(n+1+\epsilon) T_{f}(r),
$$

where. $\leq$. means the inequality holds for all $r$ outside of a set $E$ with finite Lebesgue measure, the maximum is taken over all subsets $K$ of $\{1, \ldots, q\}$ such that the linear forms $H_{j}, j \in K$, are linearly independent, and $\lambda_{H}\left(f\left(r e^{i \theta}\right)\right)$ is defined as follows:

Received by the editors February 4, 1996 and, in revised form, July 15, 1996.

1991 Mathematics Subject Classification. Primary 32A22, 32H30; Secondary 30D35.

Research supported in part by NSF grant DMS-9506424.

(C)1997 American Mathematical Society 
for each hyperplane $H=\left\{\left[x_{0}: \cdots: x_{n}\right] \in \mathbf{P}^{n}(\mathbf{C}) \mid a_{0} x_{0}+\cdots+a_{n} x_{n}=0\right\}$, denote $\|H\|=\max _{0 \leq j \leq n}\left|a_{j}\right|$ and $\|f(z)\|=\max _{0 \leq j \leq n}\left|f_{j}(z)\right|$; then

$$
\lambda_{H}\left(f\left(r e^{i \theta}\right)\right)=\log \frac{\left\|f\left(r e^{i \theta}\right)\right\|\|H\|}{H\left(f\left(r e^{i \theta}\right)\right)} .
$$

We note that the hyperplanes $H_{1}, \ldots, H_{q}$ are not assumed to lie in general position in the above theorem. By the product to sum estimate, it implies the following standard form of the Second Main Theorem for holomorphic curves.

Theorem 2.2 (SMT). Let $H_{1}, \ldots, H_{q}$ be hyperplanes in $\mathbf{P}^{n}(\mathbf{C})$ in general position. Let $f: \mathbf{C} \rightarrow \mathbf{P}^{n}(\mathbf{C})$ be a holomorphic curve whose image is not contained in any proper subspaces. Then, for any $\epsilon>0$,

$$
\sum_{j=1}^{q} m_{f}\left(H_{j}, r\right) \cdot \leq \cdot(n+1+\epsilon) T_{f}(r),
$$

where. $\leq$. means the inequality holds for all $r$ outside of a set $E$ with finite Lebesgue measure, and the proximity function $m_{f}(H, r)$ is defined as

$$
m_{f}(H, r)=\int_{0}^{2 \pi} \lambda_{H}\left(f\left(r e^{i \theta}\right)\right) \frac{d \theta}{2 \pi}
$$

In this paper, we examine the error term which appeared in Theorem 2.1, and obtain the following result:

Theorem 2.3 (A general form of the SMT with a good error term). Let $f=$ $\left[f_{0}: \cdots: f_{n}\right]: \mathbf{C} \rightarrow \mathbf{P}^{n}(\mathbf{C})$ be a holomorphic curve whose image is not contained in any proper subspaces. Let $H_{1}, \ldots, H_{q}$ be arbitrary hyperplanes in $\mathbf{P}^{n}(\mathbf{C})$. Let $\psi$ and $\phi$ be increasing functions in $\mathbf{R}^{+}$with

$$
\int_{e}^{\infty} \frac{d r}{r \psi(r)}<\infty, \text { and } \int_{e}^{\infty} \frac{d r}{\phi(r)}=\infty
$$

Then the inequality

$$
\begin{aligned}
& \int_{0}^{2 \pi} \max _{K} \sum_{k \in K} \lambda_{H_{k}}\left(f\left(r e^{i \theta}\right)\right) \frac{d \theta}{2 \pi}+N\left(R_{f}, r\right) \\
& \quad \leq(n+1) T_{f}(r)+\frac{n(n+1)}{2} \log \frac{T_{f}(r) \psi\left(T_{f}(r)\right)}{\phi(r)}+O(1)
\end{aligned}
$$

holds for all $r$ outside a set $E$ with $\int_{E} d r / \phi(r)<\infty$. Here the maximum is taken over all subsets $K$ of $\{1, \ldots, q\}$ such that the linear forms $H_{j}, j \in K$, are linearly independent, and $N\left(R_{f}, r\right)$ is the ramification term.

The error term problem was first raised by S. Lang, motivated by its analogy with number theory. Since then, a number of results have been obtained; see Lang [4], Lang-Cherry [2], Wong [17], Wong-Stoll [18] and Ye [19]. Our proof here basically repeats Ye's argument (cf. [19]).

Recall a lemma in [19],

Lemma 2.4 (Lemma 6 of [19]). Let $g$ be a non-constant meromorphic function. For arbitrary $\alpha$ with $0<\alpha l<1 / 2$, there exists a constant $C, C_{1}, C_{2}$ such that for 
any $r<\rho<R$,

$$
\frac{1}{2 \pi} \int_{0}^{2 \pi}\left|\frac{g^{(l)}\left(r e^{i \theta}\right)}{g\left(r e^{i \theta}\right)}\right|^{\alpha} d \theta \leq C\left(\frac{\rho}{r(\rho-r)}\right)^{l \alpha}\left[C_{1} T_{g}(\rho)+C_{2} \log \frac{R}{R-\rho} T_{g}(\rho)\right]^{l \alpha} .
$$

Remark. Thanks to William Cherry, (4.14) [19] is incorrect, thus, the above lemma is slightly different from Lemma 6 in [19]. To derive this lemma from Lemma 6 in [19], only (4.13) is used, which remains correct.

The following is a standard lemma in Nevanlinna theory; for reference, see [3].

Lemma 2.5. Let $\psi, \phi$ be defined in Theorem 2.3 and $T$ a non-decreasing function on $\mathbf{R}^{+}$. Then there is a constant $C>1$ such that

$$
T\left(r+\frac{\phi(r)}{\psi(T(r))}\right) \leq C T(r),
$$

for all large $r$ outside a set $E$ with $\int_{E} d r / \phi(r)<\infty$.

We now prove Theorem 2.3.

Proof. Denote by $K \subset\{1, \ldots, q\}$ such that linear forms $H_{k}, k \in K$, are linearly a set of indices independent. Without loss of generality, we may assume that $q \geq n+1$ and $\# K=n+1$. Let $T$ be the set of all injective maps $\mu:\{0,1, \ldots, n\} \rightarrow\{1, \ldots, q\}$ such that $H_{\mu(0)}, \ldots, H_{\mu(n)}$ are linearly independent. Then

$$
\begin{aligned}
& \int_{0}^{2 \pi} \max _{K} \sum_{k \in K} \lambda_{H_{k}}\left(f\left(r e^{i \theta}\right)\right) \frac{d \theta}{2 \pi} \\
& =\int_{0}^{2 \pi} \max _{\mu \in T} \sum_{j=0}^{n} \log \left(\max _{0 \leq l \leq n}\left|f_{l}\left(r e^{i \theta}\right)\right| /\left|H_{\mu(j)}\left(f\left(r e^{i \theta}\right)\right)\right|\right) \frac{d \theta}{2 \pi}+O(1) \\
& =\int_{0}^{2 \pi} \log \left\{\max _{\mu \in T}\left(\left(\max _{0 \leq l \leq n}\left|f_{l}\left(r e^{i \theta}\right)\right|\right)^{n+1} / \prod_{j=0}^{n}\left|H_{\mu(j)}\left(f\left(r e^{i \theta}\right)\right)\right|\right)\right\} \frac{d \theta}{2 \pi}+O(1) \\
& \leq \int_{0}^{2 \pi} \log \left\{\sum_{\mu \in T}\left(\left(\max _{0 \leq l \leq n}\left|f_{l}\left(r e^{i \theta}\right)\right|\right)^{n+1} / \prod_{j=0}^{n}\left|H_{\mu(j)}\left(f\left(r e^{i \theta}\right)\right)\right|\right)\right\} \frac{d \theta}{2 \pi}+O(1) \\
& =\int_{0}^{2 \pi} \log \left\{\sum_{\mu \in T} \frac{\left|W\left(H_{\mu(0)}(f), \ldots, H_{\mu(n)}(f)\right)\right|}{\left|H_{\mu(0)}(f) H_{\mu(1)}(f) \cdots H_{\mu(n)}(f)\right|}\left(r e^{i \theta}\right)\right\} \frac{d \theta}{2 \pi} \\
& +\int_{0}^{2 \pi} \log \left\{\left(\max _{0 \leq l \leq n}\left|f_{l}\left(r e^{i \theta}\right)\right|\right)^{n+1} /\left|W\left(f_{0}, \ldots, f_{n}\right)\right|\left(r e^{i \theta}\right)\right\} \frac{d \theta}{2 \pi}+O(1) .
\end{aligned}
$$

In the above, we used the property of Wronskians that

$$
\left|W\left(f_{0}, \ldots, f_{n}\right)\right|=\left|W\left(H_{\mu(0)}(f), \ldots, H_{\mu(n)}(f)\right)\right| \cdot C,
$$

where $C$ is a constant. We estimate the first term on the right-hand side of (2.2). Denote

$$
g_{\mu(l)}(z)=\frac{H_{\mu(l)}(f)}{H_{\mu(0)}(f)}(z), 0 \leq l \leq n .
$$


From the concavity of the logarithm, the Hölder inequality, Lemma 2.4 and the inequality $\left(\sum_{i, j} a_{i j}\right)^{\alpha} \leq C \sum_{i, j} a_{i j}^{\alpha}$, for $a_{i j} \geq 0$ and any $\alpha>0$, we have, for $\alpha n(n+1)<1$,

$$
\begin{aligned}
& \int_{0}^{2 \pi} \log \left\{\sum_{\mu \in T} \frac{\left|W\left(H_{\mu(0)}(f), \ldots, H_{\mu(n)}(f)\right)\right|}{\left|H_{\mu(0)}(f) H_{\mu(1)}(f) \cdots H_{\mu(n)}(f)\right|}\left(r e^{i \theta}\right)\right\} \frac{d \theta}{2 \pi} \\
& \leq \frac{1}{\alpha} \int_{0}^{2 \pi} \log \sum_{\mu \in T}\left(\frac{\left|W\left(H_{\mu(0)}(f), \ldots, H_{\mu(n)}(f)\right)\right|}{\left|H_{\mu(0)}(f) H_{\mu(1)}(f) \cdots H_{\mu(n)}(f)\right|}\left(r e^{i \theta}\right)\right)^{\alpha} \frac{d \theta}{2 \pi}+O(1) \\
& =\frac{1}{\alpha} \int_{0}^{2 \pi} \log \sum_{\mu \in T}\left(\frac{\left|W\left(1, g_{\mu(1)}, \ldots, g_{\mu(n)}\right)\right|}{\left|g_{\mu(1)} \cdots g_{\mu(n)}\right|}\left(r e^{i \theta}\right)\right)^{\alpha} \frac{d \theta}{2 \pi}+O(1) \\
& \leq \frac{1}{\alpha} \int_{0}^{2 \pi} \log \left\{\sum_{\mu \in T} \sum_{i_{1}+\cdots+i_{n} \leq n(n+1) / 2}\left|\frac{g_{\mu(1)}^{\left(i_{1}\right)}}{g_{\mu(1)}} \cdots \frac{g_{\mu(n)}^{\left(i_{n}\right)}}{g_{\mu(n)}}\right|^{\alpha}\left(r e^{i \theta}\right)\right\} \frac{d \theta}{2 \pi}+O(1) \\
& \leq \frac{1}{\alpha} \log \int_{0}^{2 \pi}\left\{\sum_{\mu \in T} \sum_{i_{1}+\cdots+i_{n} \leq n(n+1) / 2}\left|\frac{g_{\mu(1)}^{\left(i_{1}\right)}}{g_{\mu(1)}} \cdots \frac{g_{\mu(n)}^{\left(i_{n}\right)}}{g_{\mu(n)}}\right|^{\alpha}\left(r e^{i \theta}\right)\right\} \frac{d \theta}{2 \pi}+O(1) \\
& \leq \frac{1}{\alpha} \log \left\{\sum_{\mu \in T} \sum_{i_{1}+\cdots+i_{n} \leq n(n+1) / 2} \prod_{l=1}^{n}\left(\int_{0}^{2 \pi}\left|\frac{g_{\mu(l)}^{\left(i_{l}\right)}}{g_{\mu(l)}}\right|^{\alpha(n+1)}\left(r e^{i \theta}\right) \frac{d \theta}{2 \pi}\right)^{1 /(n+1)}\right\}+O(1) \\
& \leq \frac{1}{\alpha} \log \left\{\sum _ { \mu \in T } \sum _ { i _ { 1 } + \cdots + i _ { n } \leq n ( n + 1 ) / 2 } \prod _ { l = 1 } ^ { n } \left(( \frac { \rho } { r ( \rho - r ) } ) ^ { i _ { l } \alpha } \left[C_{1} T_{g_{\mu}(l)}(\rho)\right.\right.\right. \\
& \left.\left.\left.+C_{2} \log \frac{R}{\rho(R-\rho)} T_{g_{\mu(l)}}(\rho)\right]^{i_{l} \alpha}\right)\right\}+O(1) \\
& \leq \frac{n(n+1)}{2} \log \left\{\frac{\rho}{r(\rho-r)} \sum_{\mu \in T}\left[C_{1} T_{f}(\rho)+C_{2} \log \frac{R}{\rho(R-\rho)} T_{f}(\rho)\right]\right\}+O(1) .
\end{aligned}
$$

Taking

$$
R=r+\frac{\phi(r)}{\psi\left(T_{f}(r)\right)} \text { and } \rho=\frac{R+r}{2}=r+\frac{\phi(r)}{2 \psi\left(T_{f}(r)\right)},
$$

and applying Lemma 2.5 gives

$$
\begin{gathered}
T_{f}(\rho) \leq T_{f}(R) \leq C T_{f}(r), \\
\frac{R}{\rho(R-\rho)} \leq \frac{2 \Psi\left(T_{f}(r)\right)}{\phi(R)} .
\end{gathered}
$$

Recall that $\Psi$ is the function such that $\int \frac{d x}{x \Psi(x)}<\infty$, and the smaller $\Psi$, the better. So WLOG, we assume that $\Psi(x) \leq x$. Thus we have

$$
\frac{R}{\rho(R-\rho)} \leq C T_{f}(r)
$$


Thus, (2.3) becomes

$$
\begin{aligned}
& \int_{0}^{2 \pi} \log \left\{\sum_{\mu \in T} \frac{W\left(H_{\mu(0)}(f), \ldots, H_{\mu(n)}(f)\right.}{\left|H_{\mu(0)}(f) H_{\mu(1)}(f) \cdots H_{\mu(n)}(f)\right|}\left(r e^{i \theta}\right)\right\} \frac{d \theta}{2 \pi} \\
& . \leq \frac{n(n+1)}{2} \log \frac{T_{f}(r) \psi\left(T_{f}(r)\right)}{\phi(r)}+O(1) .
\end{aligned}
$$

Now

$$
\begin{aligned}
& \int_{0}^{2 \pi} \log \left\{\left(\max _{0 \leq l \leq n}\left|f_{l}\left(r e^{i \theta}\right)\right|\right)^{n+1} /\left|W\left(f_{0}, \ldots, f_{n}\right)\left(r e^{i \theta}\right)\right|\right\} \frac{d \theta}{2 \pi} \\
& =\int_{0}^{2 \pi} \log \left(\max _{0 \leq l \leq n}\left|f_{l}\left(r e^{i \theta}\right)\right|\right)^{n+1} \frac{d \theta}{2 \pi}+\int_{0}^{2 \pi} \log \frac{1}{\left|W\left(f_{0}, \ldots, f_{n}\right)\left(r e^{i \theta}\right)\right|} \frac{d \theta}{2 \pi} \\
& =(n+1) T_{f}(r)-N_{W}(0, r) \\
& =(n+1) T_{f}(r)-N\left(R_{f}, r\right) .
\end{aligned}
$$

Combining (2.2), (2.4) and (2.5), we conclude the proof.

\section{Application I: A simple proof of the Second Main Theorem FOR MOVING TARGETS}

The purpose of this section is to show how the general form of SMT with fixed targets (Theorem 2.1) implies the SMT with moving targets which was originally proved by Ru-Stoll ([8]) in 1991. We note that the usual form of the SMT with fixed targets doesn't directly imply the SMT with moving targets, but the general form does! This is one of the motivations for writing this paper. This observation is also used in another paper, joint with Vojta ([10]), to prove Schmidt's subspace theorem with moving targets in number theory.

Theorem 3.1. Let $f=\left[f_{0}: \cdots: f_{n}\right]: \mathbf{C} \rightarrow \mathbf{P}^{n}(\mathbf{C})$ be a holomorphic map. Let $\mathcal{G}$ be an arbitrary finite set of $q$ holomorphic maps $H_{j}=\left[a_{j, 0}: \cdots: a_{j, n}\right]: \mathbf{C} \rightarrow \mathbf{P}^{n}(\mathbf{C})^{*}$. Let $\mathcal{R}_{\mathcal{G}}$ be the smallest field which contains $\mathbf{C}$ and all $a_{j \mu} / a_{j \nu}$ with $a_{j \nu} \not \equiv 0$. If $f$ is non-degenerate over $\mathcal{R}_{\mathcal{G}}$, meaning that $f_{0}, \ldots, f_{n}$ are linearly independent over $\mathcal{R}_{\mathcal{G}}$, then for every $\epsilon>0$, we have

$$
\int_{0}^{2 \pi} \max _{K} \sum_{k \in K} \lambda_{H_{k}\left(r e^{i \theta}\right)}\left(f\left(r e^{i \theta}\right)\right) \frac{d \theta}{2 \pi} \cdot \leq .(n+1+\epsilon) T_{f}(r)+O\left(\max _{1 \leq j \leq q} T_{H_{j}}(r)\right),
$$

where. $\leq$. means the inequality holds except for those $r$ contained in a set $E \subset \mathbf{R}_{>0}$ of finite Lebesgue measure, and where $\max _{K}$ is taken over all subsets $K \subset\{1, \ldots, q\}$ such that $H_{k}(z)$, for $k \in K$, are linearly independent for some (and hence for almost all) $z \in \mathbf{C}$.

The moving hyperplanes $H_{1}, \ldots, H_{q}$ are said to be in general position if $H_{1}(z)$, $\ldots, H_{q}(z)$ are in general position for some (and hence for almost all) $z \in \mathbf{C}$. By the "product to sum estimate", the above theorem implies the SMT with moving targets [8]. For the sake of completeness, we recall the "product to sum estimate" lemma. 
Let $\mathcal{G}$ be a finite set of $q \geq n+1$ holomorphic maps $H_{j}=\left[a_{j, 0}: \cdots: a_{j, n}\right]: \mathbf{C} \rightarrow$ $\mathbf{P}^{n}(\mathbf{C})^{*}, 1 \leq j \leq q$, such that $H_{1}, \ldots, H_{q}$ are in general position. Define

$$
\Gamma(\mathcal{G})(z)=\min \left\{\frac{\left\|\tilde{H}_{\mu(0)}(z) \wedge \cdots \wedge \tilde{H}_{\mu(n)}(z)\right\|}{\left\|\tilde{H}_{\mu(0)}(z)\right\| \cdots\left\|\tilde{H}_{\mu(n)}(z)\right\|} \mid \mu: \mathbf{Z}[0, n] \rightarrow\{1, \ldots, q\}\right\},
$$

for all $z \in \mathbf{C}$, where $\tilde{H}_{j}=\left(a_{j, 0}, \ldots, a_{j, n}\right)$. By the general position assumption,

$$
D=\{z \in \mathbf{C} \mid \Gamma(\mathcal{G})(z)=0\}
$$

is a closed set of isolated points.

Lemma 3.2 (product to sum estimate; cf. Theorem 6.2 of $[8]$ ). Let $f=\left[f_{0}: \cdots\right.$ : $\left.f_{n}\right]: \mathbf{C} \rightarrow \mathbf{P}^{n}(\mathbf{C})$ be a holomorphic map. Let $\mathcal{G}$ be a finite set of holomorphic maps $H_{j}=\left[a_{j, 0}: \cdots: a_{j, n}\right]: \mathbf{C} \rightarrow \mathbf{P}^{n}(\mathbf{C})^{*}, 1 \leq j \leq q$, such that $H_{1}, \ldots, H_{q}$ are in general position. Take a point $z \in \mathbf{C}-D$ such that $H(f(z)) \neq 0$ for all $H \in \mathcal{G}$. Then there exist $i(z, 0), \ldots, i(z, n)$ among $1, \ldots, q$ such that

$$
\prod_{H \in \mathcal{G}} \frac{\|f(z)\|\|H(z)\|}{|H(f(z))|} \leq\left(\frac{2^{3 n+3}}{\Gamma(\mathcal{G})(z)}\right)^{q-n-1} \prod_{l=0}^{n} \frac{\|f(z)\|\left\|H_{i(z, l)}(z)\right\|}{\left|H_{i(z, l)}(f(z))\right|} .
$$

Proof. Given any $z \in \mathbf{C}-D$, we have fixed hyperplanes $\{H(z) \mid H \in \mathcal{G}\}$, and they are in general position. It is well-known (cf. Theorem 6.1 of [8]) that for the number $a=(1 / 2)^{3 n+3} \Gamma(\mathcal{G})(z)$ we have $\# I(f(z), a, \mathcal{G}) \leq n$, where

$$
I(f(z), a, \mathcal{G})=\left\{j \mid \frac{\left|H_{j}(f(z))\right|}{\|f(z)\|\left\|H_{j}(z)\right\|} \leq a, 1 \leq j \leq q\right\} .
$$

Choose $i(z, 0), \ldots, i(z, n) \in\{1, \ldots, q\}$ such that $I(f(z), a, \mathcal{G}) \subset\{i(z, 0), \ldots, i(z, n)\}$; then

$$
\begin{aligned}
\prod_{H \in \mathcal{G}} \frac{\|f(z)\|\|H(z)\|}{|H(f(z))|} & \leq\left(\frac{1}{a}\right)^{q-n-1} \prod_{l=0}^{n} \frac{\|f(z)\|\left\|H_{i(z, l)}(z)\right\|}{\left|H_{i(z, l)}(f(z))\right|} \\
& =\left(\frac{2^{3 n+3}}{\Gamma(\mathcal{G})(z)}\right)^{q-n-1} \prod_{l=0}^{n} \frac{\|f(z)\|\left\|H_{i(z, l)}(z)\right\|}{\left|H_{i(z, l)}(f(z))\right|}
\end{aligned}
$$

It is now clear that Theorem 3.1, together with Lemma 3.2, implies the SMT with moving targets.

Theorem 3.3 (SMT with moving targets; cf. [8]). Under the same assumptions in Theorem 3.1, if, in addition, we assume that the hyperplanes $H_{j}=\left[a_{j, 0}: \cdots\right.$ : $\left.a_{j, n}\right]: \mathbf{C} \rightarrow \mathbf{P}^{n}(\mathbf{C})^{*}, 1 \leq j \leq q$, are in general position, then for every $\epsilon>0$, we have

$$
\sum_{i=1}^{q} m_{f}\left(H_{i}, r\right) \cdot \leq \cdot(n+1+\epsilon) T_{f}(r)+O\left(\max _{1 \leq j \leq q} T_{H_{j}}(r)\right)
$$

where. $\leq$. means the inequality holds for all $r$ outside of a subset $E \subset \mathbf{R}_{>0}$ with finite Lebesgue measure.

We now prove Theorem 3.1. 
Proof. Without loss of generality, we can assume $q \geq n+1$, and that at least $n+1$ of the hyperplanes are linearly independent. Let $T$ be the set of all maps $\mu$ : $\{0,1, \ldots, n\} \rightarrow\{1, \ldots, q\}$ such that $H_{\mu(0)}(z), \ldots, H_{\mu(n)}(z)$ are linearly independent for some (thus for almost all) $z \in \mathbf{C}$. Without loss of generality we assume that $\left(f_{0}, \ldots, f_{n}\right)$ is a reduced representation for $f$, meaning that $f_{j}$ are entire and without common zeros. For each $1 \leq j \leq q$, choose $\bar{j}$ so that $a_{j, \bar{j}} \not \equiv 0$, and define

$$
\zeta_{j, l}(z)=a_{j, l}(z) / a_{j, \bar{j}}(z), j=1, \ldots, q ; l=0, \ldots, n .
$$

Let $\mathcal{L}(s)$ be the vector space generated over $\mathbf{C}$ by

$$
\left\{\zeta_{1,0}^{n_{1,0}} \cdots \zeta_{q, 0}^{n_{q, 0}} \cdots \zeta_{1, n}^{n_{1, n}} \cdots \zeta_{q, n}^{n_{q, n}} \mid n_{i, j} \in \mathbf{N}, \sum_{i=1}^{q} \sum_{j=0}^{n} n_{i, j}=s\right\} .
$$

We have $\mathcal{L}(s) \subset \mathcal{L}(s+1)$. Let $\left\{b_{1}, \ldots, b_{\ell(s+1)}\right\}$ be a basis of $\mathcal{L}(s+1)$ such that $\left\{b_{1}, \ldots, b_{\ell(s)}\right\}$ is a basis of $\mathcal{L}(s)$, where $\ell(s)=\operatorname{dim} \mathcal{L}(s)$. Let $F=\mathbf{P}(\tilde{F}): \mathbf{C} \rightarrow$ $\mathbf{P}\left(\mathbf{C}^{(n+1) \ell(s+1)}\right)$ be the holomorphic map defined by

$$
\begin{aligned}
\tilde{F}(z) & =\left(b_{1}(z) f_{0}(z), \ldots, b_{\ell(s+1)}(z) f_{0}(z), b_{1}(z) f_{1}(z), \ldots, b_{\ell(s+1)}(z) f_{n}(z)\right) \\
& \in \mathbf{C}^{(n+1) \ell(s+1)} .
\end{aligned}
$$

Because $f$ is linearly nondegenerate over $\mathcal{R}_{\mathcal{G}}, F(\mathbf{C})$ is not contained in any hyperplanes in $\mathbf{P}\left(\mathbf{C}^{(n+1) \ell(s+1)}\right)$. We will apply the general form of the SMT (Theorem 2.1) to $F$. The next step is to construct, for each $\mu \in T$, fixed hyperplanes $\left\{\hat{H}_{l, j}(\mu) \mid l=0, \ldots, n ; j=1, \ldots, \ell(s)\right\}$ in $\mathbf{P}\left(\mathbf{C}^{(n+1) \ell(s+1)}\right)$ such that $\lambda_{\hat{H}_{l, j}(\mu)}(F(z)) \sim$ $\lambda_{H_{\mu(l)}(z)}(f(z))$. For $j=1, \ldots, q$, let $h_{j}$ be the meromorphic function defined by

$$
h_{j}(z)=\zeta_{j, 0}+\sum_{l=1}^{n} \zeta_{j, l}(z) \frac{f_{l}(z)}{f_{0}(z)} \quad(j=1, \ldots, q) .
$$

The $b_{j} h_{\mu(l)}(j=1, \ldots, \ell(s), l=0, \ldots, n)$ can be written as $\mathbf{C}$-linear combinations of $b_{r}, 1 \leq r \leq \ell(s+1)$, and the products $b_{\alpha}\left(f_{\beta} / f_{0}\right)$ for $\alpha=1, \ldots, \ell(s+1)$ and $\beta=0, \ldots, n$. In other words there is an $(n+1) \ell(s) \times((n+1) \ell(s+1))$ matrix $C(\mu)$ with entries in $\mathbf{C}$ such that

$$
\left(\begin{array}{c}
b_{1} h_{\mu(0)} \\
\vdots \\
b_{\ell(s)} h_{\mu(0)} \\
\vdots \\
b_{1} h_{\mu(n)} \\
\vdots \\
b_{\ell(s)} h_{\mu(n)}
\end{array}\right)=C(\mu)\left(\begin{array}{c}
b_{1} \\
\vdots \\
b_{\ell(s+1)}\left(f_{1} / f_{0}\right) \\
\vdots \\
b_{\ell(s+1)}\left(f_{1} / f_{0}\right) \\
\vdots \\
b_{1}\left(f_{n} / f_{0}\right) \\
\vdots \\
b_{\ell(s+1)}\left(f_{n} / f_{0}\right)
\end{array}\right) .
$$

For $l=0, \ldots, n, j=1, \ldots, \ell(s)$, let $\hat{H}_{l, j}(\mu)$ be the hyperplane in $\mathbf{P}\left(\mathbf{C}^{(n+1) \ell(s+1)}\right)$ defined by the corresponding row in $C(\mu)$; i.e., if $c_{i j}(\mu)$ denote the elements of 
$C(\mu)$, then

$$
\begin{aligned}
\hat{H}_{l, j}(\mu) & =\left\{\left[y_{1,0}: \cdots: y_{\ell(s), 0}: y_{1,1}: \cdots: y_{\ell(s+1), 1}: \cdots: y_{1, n}: \cdots: y_{\ell(s+1), n}\right]\right. \\
& \in \mathbf{P}\left(\mathbf{C}^{(n+1) \ell(s+1)}\right) \mid c_{l \ell(s)+j, 1}(\mu) y_{1,0}+\cdots+c_{l \ell(s)+j, \ell(s+1)}(\mu) y_{\ell(s), 0} \\
& +c_{l \ell(s)+j, \ell(s+1)+1}(\mu) y_{1,1}+\cdots+c_{l \ell(s)+j, 2 \ell(s+1)}(\mu) y_{\ell(s+1), 1} \\
& \left.+\cdots+c_{l \ell(s)+j,(n+1) \ell(s+1)}(\mu) y_{\ell(s+1), n}=0\right\} .
\end{aligned}
$$

Since $H_{\mu(0)}(z), \ldots, H_{\mu(n)}(z)$ are linearly independent for some $z$ and $f_{0}, \ldots, f_{n}$ are linearly independent over $\mathcal{R}_{\mathcal{G}}, h_{\mu(0)}, \ldots, h_{\mu(n)}$ are linearly independent over $\mathcal{R}_{\mathcal{G}}$. Thus, by the choice of $b_{1}, \ldots, b_{\ell(s)},\left(b_{j} h_{\mu(l)}\right)_{j=1, \ldots, \ell(s) ; l=0, \ldots, n}$ are linearly independent over C. Hence, $\hat{H}_{l, j}(\mu), l=0, \ldots, n, j=1, \ldots, \ell(s)$, are linearly independent for each $\mu \in T$. Applying Theorem 2.1 for $F$, with the hyperplanes $\left\{\hat{H}_{l, j}(\mu) \mid l=0, \ldots, n ; j=1, \ldots, \ell(s)\right\}$, yields

$$
\int_{0}^{2 \pi} \max _{\mu \in T} \sum_{l=0}^{n} \sum_{j=1}^{\ell(s)} \lambda_{\hat{H}_{l, j}(\mu)}\left(F\left(r e^{i \theta}\right)\right) \frac{d \theta}{2 \pi} \cdot \leq .((n+1) \ell(s+1)+\epsilon / 2) T_{F}(r) .
$$

We now compare $T_{f}(r)$ and $T_{F}(r)$. In fact, for each $z \in \mathbf{C}$, not in the set of the poles of $b_{1}, \ldots, b_{\ell(s+1)}$, we have

$$
\begin{aligned}
\|\tilde{F}(z)\| \\
=\max \left(\left|b_{1}(z) f_{0}(z)\right|, \ldots,\left|b_{\ell(s+1)}(z) f_{0}(z)\right|,\left|b_{1}(z) f_{1}(z)\right|, \ldots,\left|b_{\ell(s+1)}(z) f_{n}(z)\right|\right) \\
\quad+O(1) \\
=\max \left(\left|f_{0}(z)\right|, \ldots,\left|f_{n}(z)\right|\right) \cdot \max \left(\left|b_{1}(z)\right|, \ldots,\left|b_{\ell(s+1)}(z)\right|\right) \\
=\quad\|f\| \cdot \max \left(\left|b_{1}(z)\right|, \ldots,\left|b_{\ell(s+1)}(z)\right|\right)+O(1) .
\end{aligned}
$$

By first main theorem

$$
\begin{aligned}
T_{F}(r)= & \int_{0}^{2 \pi} \log \frac{\|\tilde{F}\|}{\left|b_{1} f_{0}\right|}\left(r e^{i \theta}\right) d \theta+N\left(r,\left[b_{1} f_{0}=0\right]\right) \\
& -N\left(r,\left[1 / b_{1}=0\right]\right)+O(1) \\
= & \int_{0}^{2 \pi} \log \frac{\|f\|}{\left|f_{0}\right|}\left(r e^{i \theta}\right) d \theta+N\left(r,\left[f_{0}=0\right]\right)+O\left(\max _{1 \leq q} T_{H_{i}}(r)\right) .
\end{aligned}
$$

Therefore

$$
T_{F}(r)=T_{f}(r)+O\left(\max _{1 \leq q} T_{H_{i}}(r)\right) .
$$


Next we compare $\lambda_{H_{\mu(l)}(z)}(f(z))$ and $\lambda_{\hat{H}_{l, j}(\mu)}(F(z))$, for each $\mu \in T$. By (2.1), (3.1), (3.2), (3.3), (3.4), and (3.5)

$$
\begin{aligned}
& \lambda_{\hat{H}_{l, j}(\mu)}(F(z)) \\
& =-\log \frac{\left|c_{l \ell(s)+j, 1}(\mu) b_{1}(z) f_{0}(z)+\cdots+c_{l \ell(s)+j,(n+1) \ell(s+1)}(\mu) b_{\ell(s+1)}(z) f_{n}(z)\right|}{\max _{0 \leq r \leq n}\left|f_{r}(z)\right| \cdot \max _{1 \leq \alpha \leq \ell(s+1)}\left|b_{\alpha}(z)\right| \cdot \max _{1 \leq r \leq(n+1) \ell(s+1)}\left|c_{l \ell(s)+j, r}(\mu)\right|} \\
& =-\log \frac{\left|b_{j}(z) h_{\mu(l)}(z)\right| \cdot\left|f_{0}(z)\right|}{\max _{0 \leq r \leq n}\left|f_{r}(z)\right| \cdot \max _{1 \leq \alpha \leq \ell(s+1)}\left|b_{\alpha}(z)\right| \cdot \max _{1 \leq r \leq(n+1) \ell(s+1)}\left|c_{l \ell(s)+j, r}(\mu)\right|} \\
& =-\log \frac{\left|b_{j}(z)\right| \cdot\left|\zeta_{\mu(l), 0}(z) f_{0}(z)+\cdots+\zeta_{\mu(l), n}(z) f_{n}(z)\right|}{\max _{0 \leq r \leq n}\left|f_{r}(z)\right| \cdot \max _{1 \leq \alpha \leq \ell(s+1)}\left|b_{\alpha}(z)\right| \cdot \max _{1 \leq r \leq(n+1) \ell(s+1)}\left|c_{l \ell(s)+j, r}(\mu)\right|} \\
& =\lambda_{H_{\mu(l)}(z)}(f(z))-\log \frac{\max _{0 \leq r \leq n}\left|a_{\mu(l), r}(z)\right|}{\left|a_{\mu(l), \overline{\mu(l)}}(z)\right|}-\log \frac{\left|b_{j}(z)\right|}{\max _{1 \leq \alpha \leq \ell(s+1)}\left|b_{\alpha}(z)\right|}+O(1),
\end{aligned}
$$

where the above equality holds for those $z \in \mathbf{C}$ such that $\left\|F(z), \hat{H}_{l, j}(\mu)\right\| \neq$ $0,\left\|f(z), H_{\mu(l)}(z)\right\| \neq 0, f_{0}(z) \neq 0$, and such that $z$ is not in the union of the sets of the zeros and poles of $\zeta_{j, l}$ and $b_{r}, 1 \leq j \leq q, 0 \leq l \leq n, 1 \leq r \leq \ell(s+1)$. Combining this with (3.6) and (3.7) gives

$$
\begin{aligned}
\ell(s) & \int_{0}^{2 \pi} \max _{\mu \in T} \sum_{l=0}^{n} \lambda_{H_{\mu(l)}\left(r e^{i \theta}\right)}\left(f\left(r e^{i \theta}\right)\right) \frac{d \theta}{2 \pi} \\
= & \int_{0}^{2 \pi} \max _{\mu \in T} \sum_{l=0}^{n} \sum_{j=1}^{\ell(s)} \lambda_{\hat{H}_{l, j}(\mu)}\left(F\left(r e^{i \theta}\right)\right) \frac{d \theta}{2 \pi}+O\left(\max _{1 \leq i \leq q} T_{H_{i}}(r)\right) \\
. \leq . & ((n+1) \ell(s+1)+\epsilon / 2) T_{f}(r)+O\left(\max _{1 \leq j \leq q} T_{H_{j}}(r)\right) .
\end{aligned}
$$

Hence

$$
\begin{aligned}
& \int_{0}^{2 \pi} \max _{\mu \in T} \sum_{l=0}^{n} \lambda_{H_{\mu(l)}\left(r e^{i \theta}\right)}\left(f\left(r e^{i \theta}\right)\right) \frac{d \theta}{2 \pi} \\
& . \leq . \quad((1+n) \ell(s+1) / \ell(s)+\epsilon / 2 \ell(s)) T_{f}(r)+O\left(\max _{1 \leq j \leq q} T_{H_{j}}(r)\right) .
\end{aligned}
$$

By Steinmetz [12],

$$
\liminf _{s \rightarrow \infty} \frac{\ell(s+1)}{\ell(s)}=1 .
$$

Thus, for the given $\epsilon>0$ there exists an $s \in \mathbf{Z}_{>0}$ such that

$$
\ell(s+1) / \ell(s)<1+\epsilon / 2(n+1) .
$$

Choosing such an $s$ concludes our proof.

\section{Application 2: The SMt for linearly degenerate HOLOMORPHIC MAPPINGS WITH A GOOD ERROR TERM}

In this section we will derive the Second Main Theorem with a good error term for the holomorphic curves whose image is contained in some $k$-dimensional subspace 
of $\mathbf{P}^{n}(\mathbf{C})$. By using Nochka weights, we reduce the problem to Theorem 2.3, and so we regard this section as the second application of the general form of the Second Main Theorem (Theorem 2.3).

Theorem 4.1. Let $f=\left[f_{0}: \cdots: f_{n}\right]: \mathbf{C} \rightarrow \mathbf{P}^{n}(\mathbf{C})$ be a holomorphic map whose image is contained in some $k$-dimensional subspace but not in any subspace of dimension lower than $k$. Let $H_{j}=\left[a_{j, 0}: \cdots: a_{j, n}\right], 1 \leq j \leq q$, be hyperplanes in general position. Let $\psi$ and $\phi$ be increasing functions in $\mathbf{R}^{+}$with

$$
\int_{e}^{\infty} \frac{d r}{r \psi(r)}<\infty, \text { and } \int_{e}^{\infty} \frac{d r}{\phi(r)}=\infty
$$

Then, we have

$$
\begin{aligned}
& \sum_{i=1}^{q} m_{f, H_{i}}(r)+\left(\frac{n+1}{k+1}\right) N\left(R_{f}, r\right) \\
& \quad \leq(2 n-k+1) T_{f}(r)+\frac{k(2 n-k+1)}{2} \log \frac{T_{f}(r) \psi\left(T_{f}(r)\right)}{\phi(r)}+O(1)
\end{aligned}
$$

for all large $r$ outside a set $E$ with $\int_{E} d r / \phi(r)<\infty$.

Proof. Without loss of generality, we may assume that $f(\mathbf{C}) \subset \mathbf{P}^{k}(\mathbf{C})$. So $f: \mathbf{C} \rightarrow$ $\mathbf{P}^{k}(\mathbf{C})$ is a non-degenerate holomorphic map. We also assume that $q \geq 2 n-k+1$. Denote $\hat{H}_{j}=H_{j} \cap \mathbf{P}^{k}(\mathbf{C})$. Then $\hat{H}_{1}, \ldots, \hat{H}_{q}$ are in $n$-subgeneral position, i.e. for every set $P \subset\{1, \ldots, q\}$ with $\# P=n+1$ there are $\mu(0), \ldots, \mu(k) \in P$ such that $\hat{H}_{\mu(0)}, \ldots, \hat{H}_{\mu(k)}$ are linearly independent.

Recall the the following lemma about Nochka weights; for details, see [5], [11].

Lemma 4.2 (cf. [11]). Let $\mathcal{B}=\left\{b_{j} \in \mathbf{P}^{k}\left(\mathbf{C}^{\star}\right), 1 \leq j \leq q\right\}$ be a set of hyperplanes in $n$-subgeneral position with $2 n-k+1 \leq q$. For each $\emptyset \neq P \subset\{1, \ldots, q\}$ let $L(P)$ be the linear space generated by $\left\{\tilde{b}_{j} \mid j \in P\right\}$, where $\tilde{b}_{j}$ is a reduced representation of $b_{j}$. Then there exist a function $\omega:\{1, \ldots, q\} \rightarrow \mathbf{R}(0,1]$, called a Nochka weight, and a real number $\theta \geq 1$, called a Nochka constant, satisfying the following properties:

(i) If $j \in\{1, \ldots, q\}$, then $0<\omega(j) \theta \leq 1$.

(ii) $q-2 n+k-1=\theta\left(\sum_{j=1}^{q} \omega(j)-k-1\right)$.

(iii) If $\emptyset \neq P \subset\{1, \ldots, q\}$ with $\# P \leq n+1$, then $\sum_{j \in P} \omega(j) \leq \operatorname{dim} L(P)$.

(iv) $1 \leq(n+1) /(k+1) \leq \theta \leq(2 n-k+1) /(k+1)$.

(v) Given $E_{1}, \ldots, E_{q}$, real numbers $\geq 1$, and given any $Y \subset\{1, \ldots, q\}$ with $0<\# Y \leq n+1$, there exists a subset $M$ of $Y$ with $\# M=\operatorname{dim} L(Y)$ such that $\left\{\tilde{b}_{j}\right\}_{j \in M}$ is a basis for $L(Y)$ and

$$
\prod_{j \in Y} E_{j}^{\omega(j)} \leq \prod_{j \in M} E_{j}
$$

Similarly to the proof of Lemma 3.2 , since $H_{1}, \ldots H_{q}$ are in general position, for the number $a=(1 / 2)^{3 n+3} \Gamma(\mathcal{G})$ we have $\# I(f(z), a, \mathcal{G}) \leq n$, where

$$
I(f(z), a, \mathcal{G})=\left\{j \mid \frac{\left|H_{j}(f(z))\right|}{\|f(z)\|\left\|H_{j}\right\|} \leq a, 1 \leq j \leq q\right\} .
$$


Choose $i(z, 0), \ldots, i(z, n) \in\{1, \ldots, q\}$ such that $I(f(z), a, \mathcal{G}) \subset\{i(z, 0), \ldots, i(z, n)\}$, and then

$$
\prod_{j=1}^{q}\left(\frac{\|f(z)\|\left\|H_{j}\right\|}{\left|H_{j}(f(z))\right|}\right)^{\omega(j)} \leq\left(\frac{1}{a}\right)^{\sum_{j \neq i(z, l)} \omega(j)} \prod_{l=0}^{n}\left(\frac{\|f(z)\|\left\|H_{i(z, l)}\right\|}{\left|H_{i(z, l)}(f(z))\right|}\right)^{\omega(i(z, l))}
$$

where $\omega(j)$ is the Nochka weight corresponding to $\hat{H}_{j}$. Applying Lemma 4.2 with $E_{l}=e^{\lambda_{\hat{H}_{i(z, l)}}(f(z))}, 0 \leq l \leq n$, there is a subset $M$ of $Y=\{i(z, 0), \ldots, i(z, n)\}$ with $\# M=k+1$ such that $\left\{\hat{H}_{i(z, j)} \mid i(z, j) \in M\right\}$ is linearly independent, and

$$
\prod_{l=0}^{n} e^{\omega(i(z, l)) \lambda_{\hat{H}_{i(z, l)}}(f(z))} \leq \prod_{i(z, j) \in M} e^{\lambda_{\hat{H}_{i(z, j)}}(f(z))}
$$

Thus

$$
\sum_{l=0}^{n} \omega(i(z, l)) \lambda_{\hat{H}_{i(z, l)}}(f(z)) \leq \sum_{i(z, j) \in M} \lambda_{\hat{H}_{i(z, j)}}(f(z)) \leq \max _{\gamma \in \Gamma} \sum_{l=0}^{k} \lambda_{\hat{H}_{\gamma(l)}}(f(z)),
$$

where $\Gamma$ is the set of all maps $\gamma:\{0, \ldots, k\} \rightarrow\{1, \ldots, q\}$ such that $\hat{H}_{\gamma(0)}, \ldots, \hat{H}_{\gamma(k)}$ are linearly independent. Combining with (4.1) gives

$$
\sum_{j=1}^{q} \omega(j) m_{f}\left(H_{j}, r\right) \leq \int_{0}^{2 \pi} \max _{\gamma \in \Gamma} \sum_{l=0}^{k} \lambda_{\hat{H}_{\gamma(l)}}\left(f\left(r e^{i \theta}\right)\right) \frac{d \theta}{2 \pi}+O(1) .
$$

Applying Theorem 2.3 yields

$$
\begin{aligned}
& \int_{0}^{2 \pi} \max _{\gamma \in \Gamma} \sum_{l=0}^{k} \lambda_{\hat{H}_{\gamma(l)}}\left(f\left(r e^{i \theta}\right)\right) \frac{d \theta}{2 \pi} \\
& . \leq . \quad(k+1) T_{f}(r)-N\left(R_{f}, r\right)+\frac{k(k+1)}{2} \log \frac{T_{f}(r) \psi\left(T_{f}(r)\right)}{\phi(r)}+O(1) .
\end{aligned}
$$

Therefore

$$
\begin{aligned}
\sum_{j=1}^{q} \omega(j) m_{f}\left(H_{j}, r\right) \cdot \leq & (k+1) T_{f}(r)-N\left(R_{f}, r\right) \\
& +\frac{k(k+1)}{2} \log \frac{T_{f}(r) \psi\left(T_{f}(r)\right)}{\phi(r)}+O(1) .
\end{aligned}
$$

Combining this with Lemma 4.2, and recalling that $m_{f}\left(H_{j}, r\right) \leq T_{f}(r)+O(1)$, we have 


$$
\begin{aligned}
\sum_{j=1}^{q} m_{f}\left(H_{j}, r\right)= & \sum_{j=1}^{q}(1-\theta \omega(j)) m_{f}\left(H_{j}, r\right)+\sum_{j=1}^{q} \theta \omega(j) m_{f}\left(H_{j}, r\right) \\
. \leq & \sum_{j=1}^{q}(1-\theta \omega(j)) m_{f}\left(H_{j}, r\right)+\theta(k+1) T_{f}(r)-\theta N\left(R_{f}, r\right) \\
& +\theta \frac{k(k+1)}{2} \log \frac{T_{f}(r) \psi\left(T_{f}(r)\right)}{\phi(r)}+O(1) \\
\leq & \sum_{j=1}^{q}(1-\theta \omega(j)) T_{f}(r)+\theta(k+1) T_{f}(r)-\left(\frac{n+1}{k+1}\right) N\left(R_{f}, r\right) \\
& +\frac{(2 n-k+1) k}{2} \log \frac{T_{f}(r) \psi\left(T_{f}(r)\right)}{\phi(r)}+O(1) \\
= & \left\{q-\theta\left(\sum_{1 \leq j \leq q} \omega(j)-k-1\right)\right\} T_{f}(r)-\left(\frac{n+1}{k+1}\right) N\left(R_{f}, r\right) \\
& +\frac{(2 n-k+1) k}{2} \log \frac{T_{f}(r) \psi\left(T_{f}(r)\right)}{\phi(r)}+O(1) \\
= & (2 n-k+1) T_{f}(r)-\left(\frac{n+1}{k+1}\right) N\left(R_{f}, r\right) \\
& +\frac{(2 n-k+1) k}{2} \log \frac{T_{f}(r) \psi\left(T_{f}(r)\right)}{\phi(r)}+O(1) .
\end{aligned}
$$

\section{ACKNOWLEDGEMENTS}

I am grateful to the referee for pointing out a gap in the earlier version of this paper, and for his/her many helpful comments and constructive suggestions. I also wish to thank the MSRI, Berkeley, for kind hospitality during which part of the work on this paper took place.

\section{REFERENCES}

1. H. Cartan, Sur les zéros des combinaisions linéaires de p fonctions holomorpes données, Mathematica (Cluj) 7 (1933), 80-103.

2. S. Lang and W. Cherry, Topics in Nevanlinna theory, Lect. Notes Math. 1433, Springer-Verlag, Berlin, 1990. MR 91k:32025

3. W. Hayman, Meromorphic functions, Clarendon Press, 1964. MR 29:1337

4. S. Lang, The error term in Nevanlinna theory, II. Bull. of the AMS, 22 (1990), 115-126. MR 90k:32080

5. E.I. Nochka, Defect relations of meromorphic curves (in Russian), Izv. Moldavian Acad. Sc. for Phy. \& Math. Sc. 1982, no.1, 1 (1982), 41-47. MR 84g:32039

6. E.I. Nochka, On the theory of meromorphic curves, Sov. Math. Dokl. 27(2) (1983), 377-381. MR 85i: 32038

7. C. F. Osgood, Sometimes effective Thue-Siegel-Roth-Schmidt-Nevanlinna bounds or better, J. Number Theory 21 (1985), 347-389. MR 87f:11046

8. M. Ru and W. Stoll, The second main theorem for moving targets, The Journal of Geometric Analysis, 1 (1991), No. 2, 99-138. MR 92j:32098

9. M. Ru and W. Stoll, The Cartan conjecture for moving targets, Proceedings of Symposia in Pure Mathematics, American Math. Society, Vol 52 (1991), Part 2, 477-508. MR 93f:32028

10. M. Ru and P. Vojta, Schmidt's subspace theorem with moving targets, Invent. Math. 127 (1997), 51-65. CMP 1997\#5 
11. M. Ru and P. M. Wong, Integeral points of $P^{n}-\{2 n+1$ hyperplanes in general position $\}$, Invent. Math., 106 (1991), 195-216. MR 93f:11056

12. N. Steinmetz, Eine Verallgemeinerung des zweiten Nevanlinnaschen Hauptzatzes, J. Reine Angew. Math. 368 (1986), 134-141. MR 87i:30056

13. W. Stoll, An extension of the theorem of Steinmetz-Nevanlinna to holomorphic curves, Math. Ann., 282 (1988), 185-222. MR 89m:30064

14. P. Vojta, Diophantine approximation and value distribution theory, Lect. Notes Math. 1239, Springer-Verlag, Berlin etc., 1987. MR 91k:11049

15. P. Vojta, On Cartan's theorem and Cartan's conjecture, Amer. J. Math. 119 (1997), 1-17. CMP 1997\#6

16. P. Vojta, Roth's theorem with moving targets, International Mathematics Research Notices, (1996), 109-114. MR 96k:11087

17. P.M. Wong, On the second main theorem of Nevanlinna theory, Amer. J. Math., 111 (1989), 549-583. MR 91b:32030

18. P.M. Wong and W. Stoll, Second main theorem of Nevanlinna theory for non-equidimensional meromorphic maps, Amer. J. Math., 116 (1994), 1031-1071. MR 95g:32042

19. Z. Ye, On Nevanlinna's second main theorem in projective space, Invent. Math., 122 (1995), 475-507. MR 96j:32030

Department of Mathematics, University of Houston, Houston, Texas 77204

E-mail address: minru@math.uh.edu 